\title{
TWISTED FREE TENSOR PRODUCTS
}

BY

ELYAHU KATZ

\begin{abstract}
A twisted free tensor product of a differential algebra and a free differential algebra is introduced. This complex is proved to be chain homotopy equivalent to the complex associated with a twisted free product of a simplicial group and a free simplicial group. In this way we turn a geometric situation into an algebraic one, i.e. for the cofibration $Y \rightarrow Y$ $\cup_{g} C X \rightarrow \Sigma X$ we obtain a spectral sequence converging into $H(\Omega(Y$ $\left.\cup_{g} C X\right)$ ). The spectral sequence obtained in the above situation is similar to the one obtained by $L$. Smith for a cofibration. However, the one we obtain has more information in the sense that differentials can be traced, requires more lax connectivity conditions and does not need the ring of coefficients to be a field.
\end{abstract}

1. Introduction. E. H. Brown [2] replaced the complex of the total space of a fiber bundle by a twisted tensor product of the complex of the fiber and the complex of the base space. Using Gugenheims approach to this theorem in a simplicial category [3], we replace the complex of the total space of a principal cofiber bundle by a twisted free tensor product of the complexes of the cobase and cofiber (for definitions see $\$ \$ 2$ and 3). Thus we convert a geometric problem to an algebraic one. This is done by the method of acyclic models. The complex obtained yields a spectral sequence which under some connectivity conditions provides us with a converging spectral sequence where the first terms and differentials, depending on the degree of connectivity, are computable. Under quite mild connectivity conditions we obtain a spectral sequence converging to $H\left(\Omega\left(Y \cup_{g} C X\right)\right)$, which especially apply to computing the homology of the loop space of a space with cells attached to it. The spectral sequence mentioned above is similar to the spectral sequence of the cofibration $Y \rightarrow Y \cup_{8} C X \rightarrow \Sigma X$ obtained by L. Smith [9] by the aid of the cobar construction. A similar approach to that of [9] was taken by R. C. Vile in his Cornell University unpublished thesis. However, our conditions are milder and provide more information.

My interest in this paper is thanks to I. Berstein who suggested that I compute the differentials of the spectral sequence of a cofibration obtained by L. Smith [9]. I rely mainly on Gugenheim's approach [3] to a theorem of E. H. Brown [2]. Spaces and maps are in the simplicial set category, groups and

Presented to the Society, August 11, 1978; received by the editors March 22, 1978 and, in revised form, January 11, 1979.

AMS (MOS) subject classifications (1970). Primary 55F99; Secondary 55J10.

Key words and phrases. Principal cofiber bundle, twisted tensor product, twisted free tensor product. 
homomorphisms are in the category of simplicial groups.

2. Principal cofiber bundles. In this section we recall the definition of a principal cofiber bundle (p.c.b.) [8], and form a category out of p.c.b.s. We shall focus on the morphisms of this category, an approach which was not taken originally. We also recall the definitions of a twisted cartesian product (t.c.p.) [3], [7] and a twisted free product (t.f.p.) [8]. We relate t.c.p.s and t.f.p.s with each other and with p.c.b.s.

To define a p.c.b. we need the notions of a free product of groups and a cogroup. Let $G_{1}$ and $G_{2}$ be groups. Their free product $G_{1} * G_{2}$ is a group such that $\left(G_{1} * G_{2}\right)_{n}=\left(G_{1}\right)_{n} *\left(G_{2}\right)_{n}$, and the boundary and degeneracy operators are homomorphisms induced by the boundary and degeneracy operators of $G_{1}$ and $G_{2}$ respectively. Let $F X$ be the Milnor free group construction on the space $X . F X$ is defined as follows: $(F X)_{n}$ is the free group generated by $X_{n}$ and the boundary and degeneracy operators are induced by those of $X$. If $X_{0}$ has a single element $x_{0}$ then we add to $(F X)_{n}$ the single relation that identifies the degeneration of $x_{0}$ in $X_{n}$ with the identity. We set $\bar{\phi}: F X \rightarrow F^{1} X * F^{2} X$ the homomorphism induced by the map which sends $x \in X$ to ${ }^{1} x \cdot{ }^{2} x$, where ${ }^{2} X$ and ${ }^{2} X$ are copies of $X$, and ${ }^{1} x,{ }^{2} x$ correspond to $x$ in ${ }^{1} X,{ }^{2} X$ respectively. $[F X, \phi]$ is called a cogroup and $\bar{\phi}$ is its comultiplication [4]. We are now ready for the definition of a p.c.b.

A p.c.b. $[A, T, F X, \phi]$ is a homomorphism $\phi: T \rightarrow T * F X$ where $A$ is a subgroup of $T$ consisting of the elements $\{a \in T \mid \phi(a) \in T \subset T * F X\}$ and is called the cobase. The group $T$ is called the total space, and the cogroup $F X$ is called the cofiber. The co-operation $\phi$ must satisfy:

(i) $\left(1_{T} * \bar{\phi}\right) \phi=\left(\phi * 1_{F X}\right) \phi$,

(ii) $r_{1} \phi=1_{T}$,

(iii) $r_{2} \phi$ is onto,

where $1_{T}, 1_{F X}$ are the identities of $T, F X$, and $r_{1}, r_{2}$ are the projection homomorphisms from $T * F X$ to $T, F X$ respectively.

A morphism $[g, h, F k]:[A, T, F X, \phi] \rightarrow\left[A^{\prime}, T^{\prime}, F X^{\prime}, \phi^{\prime}\right]$ is a homomorphism $h: T \rightarrow T^{\prime}$ which restricts to the homomorphism $g: A \rightarrow A^{\prime}$ and makes the following diagram commutative:

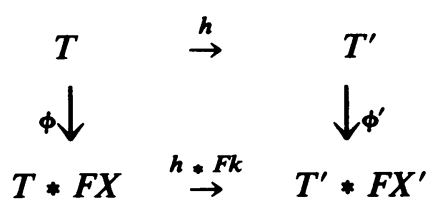

where $F k: F X \rightarrow F X^{\prime}$ is the homomorphism induced by the map $k: X \rightarrow X^{\prime}$.

Before going into the definitions of a t.c.p. and t.f.p. we have to introduce another concept.

A twisting function $t: X \rightarrow A$ is a function of degree -1 from a space to a group which honors the identities:

(i) $\partial_{0} t(x)=t\left(\partial_{1} x\right) \cdot t\left(\partial_{0} x\right)^{-1}$,

(ii) $\partial_{i} t(x)=t\left(\partial_{i+1} x\right), i>0$, 
(iii) $s_{i} t(x)=t\left(s_{i+1} x\right), i>0$,

(iv) $t\left(s_{0} x\right)=e$.

A t.c.p. $A \times, X$ is a cartesian product of a group $A$ and a space $X((A$ $\left.X_{1} X\right)_{n}=A_{n} \times X_{n}$ ) with the face and degeneracy operators:

(i) $\partial_{0}(a, x)=\left(\partial_{0} a t(x), \partial_{0} x\right)$,

(ii) $\partial_{i}(a, x)=\left(\partial_{i} a, \partial_{i} x\right), i>0$,

(iii) $s_{i}(a, x)=\left(s_{i} a, s_{i} x\right), i>0$,

where $t$ is a twisting function.

A morphism [ $g, k]: A \times, x \rightarrow A^{\prime} \times_{t^{\prime}} X^{\prime}$ is a map which sends $(a, x)$ to $(g(a), k(x))$, where the homomorphism $g$ and map $k$ make the diagram commutative:

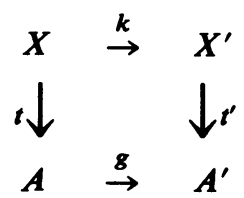

A t.f.p. $A *, F X$ with twisting function $t$ is the free product of the groups $A$ and $F X$. If $\partial_{i}^{A}, s_{i}^{A}, \partial_{i}{ }^{F X}, s_{i}^{F X}$ are the boundary and degeneracy operators of $A$ and $F X$ respectively, then we define the operations $\partial_{i}, s_{i}$ of $A *{ }_{i} F X$ as follows:

(i) $\partial_{0}$ is induced by the homomorphism $\partial_{0}^{A}$ and the homomorphism from $F X$ to $A * F X$ which is defined by the map which sends $x \in X$ to $t(x) \partial_{0} x$ in $A * F X$.

(ii) $\partial_{i}=\partial_{i}^{A} * \partial_{i}^{F X}, i>0$.

(ii) $s_{i}=s_{i}^{A} * s_{i}^{F X}, i>0$.

A morphism $[g, F k]: A *, F X \rightarrow A^{\prime} *{ }^{\prime} F X^{\prime}$ is a homomorphism induced by the homomorphisms $g$ and $F(k)$, where $g$ and $k$ satisfy the same conditions as for $[g, k]$ to be a morphism in the category of t.c.p.s [see above].

For future needs, we point out that if $A *{ }_{t} F X$ is a t.f.p. and $B$ is a group, we can produce a closely related t.f.p. $(B * A) *{ }_{i} F X$, where $\bar{t}: X \rightarrow B * A$, the twisting function, is the composition of $t$ and the inclusion homomorphism of $A$ into $B * A$. It is easy to confirm that $\bar{t}$ is a twisting function.

In light of the above definitions, there is an obvious relation between t.c.p.s and t.f.p.s.

LemMa 1. The functor which sends $A \times, X$ to $A *$, $F X$, and $[g, k]$ to $[g, F k]$ is an isomorphism.

The relation between p.c.b.s and t.f.p.s is much more complicated and is stated next.

TheOREM [8, TheOREM 2.2.3]. The total space of a p.c.b. $[A, T, F X, \phi]$ is isomorphic to a t.f.p. $A *, F X$, with some twisting function $t$. Moreover $\phi$ can be expressed as the homomorphism $1_{A} * \bar{\phi}$.

We note that the above theorem does not provide us with a canonical 
correspondence from p.c.b.s to t.f.p.s. The total space of a p.c.b. may have more than one representation as a t.f.p.

3. The construction of a twisted free tensor product. In this section we associate with every t.f.p. $A *, F X$, a differential graded algebra, which we call a twisted free tensor product (t.f.t.p.). To do this we require $X$ to be reduced (i.e. $X$ has a single element in dimension 0 ). Thus from here to the end of this paper $X$ will always stand for a reduced space. We also denote by $\boldsymbol{R}$ a fixed commutative ring with unit, and all complexes are over this ring. Before going into the construction of a t.f.t.p., we need some preliminaries.

Let $R(X)$ be the normalized chain complex associated with the simplicial set $X$, and let $\psi: R(X) \rightarrow R(X) \otimes R(X)$ be the chain map associated with the diagonal map of $X$. This map turns $R(X)$ into an augmented differential graded coalgebra. Similarly, if $A$ is a simplicial group, the multiplication of $A$ induces a map $m: R(A) \otimes R(A) \rightarrow R(A)$ which turns $R(A)$ into an augmented differential graded algebra.

For a twisting function $t: X \rightarrow A$, a twisting cochain $f_{t}: R(X) \rightarrow R(A)$ is introduced. This cochain can also be extended to a chain map between the unnormalized chain complexes of $X$ and $A$, by sending degenerate elements to 0 . This extended chain map will be used in the proof of Lemma 4. We will be using the following properties of $f_{t}$, extensively.

If $[g, k]: A \times, X \rightarrow A^{\prime} \times t^{\prime} X^{\prime}$ is a map, then the following diagram commutes:

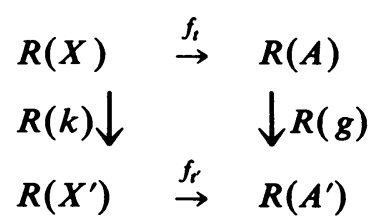

If $x \in X_{1}$ then $f_{t}(x)=1-t_{(x)}^{-1}$.

For more details see $[3,3.1,7.6,8.1]$ or $[7, \S 3]$.

Let $K$ be a differential graded chain complex with augmentation. Let $\tilde{K}$ denote its augmentation. The free tensor algebra generated by $K$ is

$$
T(K)=R \oplus \sum_{i=1}^{\infty} \tilde{K}^{i}, \quad \tilde{K}^{i}=\tilde{K} \otimes \cdots \otimes \tilde{K} \quad(n \text { times }),
$$

where the algebra multiplication is induced by the isomorphisms $\tilde{K}^{i} \otimes \tilde{K}^{j} \approx$ $\tilde{K}^{i+j}$, the differential is the derivation induced by the differential of $K$, and the augmentation is the obvious one.

Note that a derivation on a graded algebra $U$ is a function $\partial: U \rightarrow U$ of degree -1 which satisfies the following equation for homogenous elements $a, b \in U:$

$$
\partial(a b)=a \cdot b+(-1)^{\operatorname{deg} a} a \cdot \partial b .
$$

Let $U_{1}, \ldots, U_{r}$ be differential graded augmented algebras. Their coproduct is defined by: 


$$
U_{1} \amalg \cdots \amalg U_{r}=R \oplus \sum_{J} \tilde{U}_{J}
$$

where $J$ exhausts the collection of all sequences alternating on $1, \ldots, r$. If $J=\left(j_{1}, j_{2}, \ldots, j_{n}\right)$, then $\tilde{U}_{J}=\tilde{U}_{j_{1}} \otimes \cdots \otimes \tilde{U}_{j_{n}}$. The algebra product in $U_{1} \amalg \cdots \amalg U_{r}$ is induced by the algebra structures of $U_{i}$ and the tensor product. The differential is the derivation induced by the differentials of $U_{i}$, $1<i<r[1]$.

The t.f.t.p. $R(A) \amalg_{f_{t}} T(R(X))$ associated with $A *{ }_{t} F X$ is $R(A) \amalg T(R(X))$ with differential $D=D_{0}+D_{f} . D_{0}$ is the derivation induced by the differentials of $R(A)$ and $T\left(R(X)\right.$ ), which is again a differential (i.e. $D_{0}^{2}=0$ ). $D_{f}$ is the derivation defined on the algebra generators of $R(A) \amalg T(R(X))$ as follows:

$$
D_{f_{t}}(y)= \begin{cases}0 & \text { if } y \in R(A), \\ \left(f_{t} \otimes 1_{R(X)}\right) \psi(y) & \text { if } y \in R(X) .\end{cases}
$$

It is obvious that the sum of two derivations is a derivation. Thus $D=D_{0}+$ $D_{f}$ is also a derivation. We prove:

LemMa 2. $D$ is a differential (i.e. $D^{2}=0$ ).

Proof. Since $D$ is a derivation, it is enough to show that $D^{2}=0$ on algebra generators. It is obvious that $D^{2}(y)=0$ for $y \in R(A)$. We have to show that $D^{2}(y)=0$ for $y \in(R(X))_{n}$. At this point it is convenient to introduce additional notation. Let $x \in R(X)$ and $a \in R(A)$, we define:

$$
f_{t} \cap(a \otimes x)=\left(m \otimes 1_{R(X)}\right)\left(1_{R(A)} \otimes f_{t} \otimes 1_{R(X)}\right)\left(1_{R(A)} \otimes \psi\right)(a \otimes x) .
$$

In this notation $D_{f}(x)=f_{t} \cap(1 \otimes x)$. Since $f_{t}$ is a twisting cochain, the statement that $D_{f_{i}}^{2}(x)=0$, with some minor notational changes, is just the statement of Lemma 3.1 of [3]. This completes the proof.

With the multiplication of $R(A) \amalg T(R(X))$ it is readily seen that:

LEMMA 3. The complex $R(A) \amalg_{f_{i}} T(R(X))$ is an augmented differential graded algebra.

4. The main result. In this section we prove the following chain homotopy equivalence:

$$
R\left(A *{ }_{t} F X\right) \cong R(A) \amalg_{f_{i}} T(R(X)) .
$$

The proof is based on the method of acyclic models, see for example [3, §4]. Thus we next define a category and models.

The objects of the category are tuples of t.f.p.s. A morphism is a tuple of homomorphisms

$$
\begin{aligned}
\left(h_{1}^{j_{1}}, \ldots, h_{k}^{j_{k}}\right): & \left(A_{1} *_{t_{1}} F X_{1}, \ldots, A_{k} *{ }_{t_{k}} F X_{k}\right) \\
& \rightarrow\left(A_{1}^{\prime} *{ }_{t_{1}^{\prime}} F X_{1}^{\prime}, \ldots, A_{l}^{\prime} *{ }_{t_{i}^{\prime}} F X_{l}^{\prime}\right)
\end{aligned}
$$

where $h_{1}^{j}: A_{i} *_{t_{i}} F X_{i} \rightarrow A_{j_{i}}^{\prime}{ }_{t_{j_{i}}} F X_{j_{i}}^{\prime}, 1<i<k, 1<j_{1}<l$ are morphisms in the category of t.f.p.s. The composition of morphisms in the category are defined in the obvious way. To define the models we need Kan's construc- 
tion, which is also the equivalent of the loop space construction in the category of topological spaces.

For a reduced simplicial set $K$, the Kan construction $G K$ is defined as follows: $(G K)_{n}$ is the group generated by the elements [ $k$ ] for each $k \in K_{n+1}$, with the relations $\left[s_{0}(l)\right]=1$ for each $l \in K_{n}$. The face and degeneracy operators are defined by:

(i) $\partial_{0}[k]=\left[\partial_{0} k\right]^{-1}\left[\partial_{1} k\right]$,

(ii) $\partial_{i}[k]=\left[\partial_{i+1} k\right], i>0$,

(iii) $s_{i}[k]=\left[s_{i+1} k\right], i>0$.

We denote by $\tau$ the twisting function $\tau: K \rightarrow G K$ which is defined as follows: $\tau(k)=[k]^{-1}, k \in K_{n+1}$.

The models in the category of tuples will be denoted as follows:

$$
\left(M_{i_{1}}^{j_{1}}, \ldots, M_{i_{k}}^{j_{k}}\right)=\left(F \Delta_{j_{1}} * G \bar{\Delta}_{i_{1}} * F \bar{\Delta}_{i_{1}}, \ldots, F \Delta_{j_{k}} * G \bar{\Delta}_{i_{k}} * F \bar{\Delta}_{i_{k}}\right)
$$

Where $\Delta_{i}$ is the standard simplex with only one nondegenerate element of dimension $i, \bar{\Delta}_{i}$ is obtained by identifying the elements of dimension 0 , and $\bar{\tau}$ : $\bar{\Delta}_{i} \rightarrow F \Delta_{j} * G \bar{\Delta}_{i}$ is the composition of $\tau: \bar{\Delta}_{i} \rightarrow G \bar{\Delta}_{i}$ as defined above, and the inclusion homomorphism $G \bar{\Delta}_{i} \rightarrow F \Delta_{j} * G \bar{\Delta}_{i}$.

We define two functors $\alpha, \beta$. On the objects they are defined by:

$$
\begin{gathered}
\alpha\left(A_{1} *{ }_{t_{1}} F X_{1}, \ldots, A_{s} *{ }_{t_{s}} F X_{s}\right)=R\left(A_{1} *{ }_{t_{1}} F X_{1} * \cdots * A_{s} *{ }_{t_{s}} F X_{s}\right), \\
\beta\left(A_{1} *{ }_{t_{1}} F X_{1}, \ldots, A_{s} *{ }_{t_{s}} F X_{s}\right) \\
\quad=R\left(A_{1}\right) \amalg_{f_{t_{1}}} T\left(R\left(X_{1}\right)\right) \amalg \cdots \amalg R\left(A_{s}\right) \amalg_{f_{t_{s}}} T\left(R\left(X_{s}\right)\right),
\end{gathered}
$$

and on the morphisms they are defined by:

$$
\begin{aligned}
& \alpha\left(h_{1}, \ldots, h_{s}\right)=R\left(h_{1} * \cdots * h_{s}\right), \\
& \beta\left(h_{1}, \ldots, h_{s}\right)=\beta\left(h_{1}\right) \amalg \cdots \amalg\left(h_{s}\right) .
\end{aligned}
$$

If $h_{i}^{j}: A_{i}{ }^{*}{ }_{i} F X_{i} \rightarrow A_{j}{ }^{*}{ }_{j} F X_{j}$ is a morphism, then $h_{i}^{j}$ can be expressed as $h_{i}^{j}=[g, F k]$, for a homomorphism $g: A_{i} \rightarrow A_{j}$ and a map $k: x_{i} \rightarrow x_{j}$. The morphism

$$
\beta\left(h_{i}^{j}\right): R\left(A_{i}\right) \amalg_{f_{i}} T\left(R\left(X_{i}\right)\right) \rightarrow R\left(A_{j}\right) \amalg_{f_{i j}} T\left(R\left(X_{j}\right)\right)
$$

is the algebra homomorphism induced by $R(g): R\left(A_{i}\right) \rightarrow R\left(A_{j}\right)$ and $R(k)$ : $R\left(X_{i}\right) \rightarrow R\left(X_{j}\right)$.

LEMMA 4. The functors $\alpha$ and $\beta$ are representable.

Proof. In the proof of this lemma only, we assume that the complexes are unnormalized. However, because of Lemma 29.5 of [7] the result will also hold for the normalized case.

Let $a \in A_{m} \subset A *{ }_{t} F X$. We define a map

$$
[v, u]: F \Delta_{m} * G \bar{\Delta}_{m} *{ }_{\bar{\tau}} F \bar{\Delta}_{m} \rightarrow A *{ }_{t} F X,
$$

where $v: F \Delta_{m} * G \bar{\Delta}_{m} \rightarrow A$ is the homomorphism induced by the homomor- 
phisms $\tilde{a}: F \Delta_{m} \rightarrow A$ and $\tilde{e}: G \Delta_{m} \rightarrow A, u: F \bar{\Delta}_{m} \rightarrow F X$ is the constant homomorphism. The homomorphism $\tilde{a}$ is the one that sends $\delta_{m}$ (the only nondegenerate element of dimensions $m$ in $\Delta_{m}$ ) to $a$, and $\tilde{e}$ is the constant homomorphism. We readily have $\alpha[v, u]\left(\delta_{m}\right)=a$. For $x \in X_{m} \subset A *{ }_{t} F X$ we define a map

$$
\left[v^{\prime}, u^{\prime}\right]: G \bar{\Delta}_{n} *{ }_{\tau} F \bar{\Delta}_{n} \rightarrow A *{ }_{t} F X,
$$

where $u^{\prime}=F \tilde{x}$ is the homomorphism induced by the map $\tilde{x}: \bar{\Delta}_{n} \rightarrow X$ which sends $\delta_{m}$ to $x, v^{\prime}$ is the composite of the homomorphisms $G \tilde{x}: G \bar{\Delta}_{n} \rightarrow G X$ and $G_{t}: G X \rightarrow A$ induced by the maps $\tilde{x}$ and $t$. It is easy to confirm that $\alpha\left[v^{\prime}, u^{\prime}\right]\left(\delta_{n}\right)=x$. The treatment of $x^{-1} \in F X\left(x \in X_{n}\right)$ is done in the same way $x$ was treated except that $\tilde{x}$ is replaced by $\tilde{x}^{-1}$.

At this point, the use of the category of tuples comes in. We have to show the representability of generators, which are elements of the form:

$$
y_{1} \cdots y_{r} \in R\left(A_{1} *{ }_{t_{1}} F X_{1} * \cdots * A_{s} * t_{s} F X_{s}\right)
$$

where $y_{i} \in \cup_{l=1}^{s}\left(A_{l} \cup X_{l} \cup X_{l}^{-1}\right), 1<i<r$, are homogenous elements, no two adjacent $y_{i}$ 's are in a fixed $A_{l}$, and $X_{l}^{-1}$ is a copy of $X_{l}$ whose elements correspond to the inverses of $X_{l}$ in $F X_{l}$. For each $y_{k}, 1<k<r$, we obtain in the above way a t.c.p. $M_{i_{k}}^{j_{k}}$ and a homomorphism $h_{k}$. Then $\left(M_{i_{1}}^{j_{1}}, \ldots, M_{i_{k}}^{j_{r}}\right)$ and $\left(h_{1}, \ldots, h_{r}\right)$ with the proper element in $\alpha\left(M_{i_{1}}^{j_{1}}, \ldots, M_{i_{r}}^{j_{r}}\right)$ give us a representation for $y_{1} \cdots y_{r}$. Note that even if $s=1$, we may have $r>1$.

The representability of $\beta$ is proved similarly. Let $\alpha \in(R(A))_{n} \subset R(A)$ $\amalg_{f_{i}} T(R(X))$, and $x \in(R(X))_{n} \subset R(A) \amalg_{f_{i}} T(R(X))$ be the same elements of the first part of the proof. Then $\beta[v, u]\left(\delta_{m}\right)=a$ and $\beta\left[v^{\prime}, u^{\prime}\right]\left(\delta_{n}\right)=x$, and we proceed as in the previous case.

LEMMA 5. The functors $\alpha$ and $\beta$ are acyclic on the models. (By acyclicity, we mean that homology applied on the functors is $R$ in dimension 0 and 0 in dimensions larger than 0.)

Proof. It is enough to prove the acyclicity of $\alpha\left(M_{i}^{j}\right)$ and $\beta\left(M_{i}^{j}\right)$. To show this we make the following remark. If $U_{1}, \ldots, U_{n}$ are acyclic differential graded augmented algebras then:

$$
U_{1} \amalg \cdots \amalg U_{n}=R \oplus \sum_{J} U_{J} \quad \text { (as defined in §3) }
$$

is also acyclic. This follows by applying the Künneth formula to each of the $U_{J}$, and the fact that homology commutes with direct sums.

Now if $\beta\left(M_{i}^{j}\right), i, j>0$, are acyclic, by the last remark it follows directly that $\beta$ is acyclic on the models. If $\alpha\left(M_{i}^{j}\right), k, j>0$, are acyclic, the acyclicity of $\alpha$ on the models follows from the remark and Theorem 1 of [6] which states:

Let $G_{1}, G_{2}$ be groups then we have a homotopy chain equivalence: $R\left(G_{1} * G_{2}\right) \simeq R\left(G_{1}\right) \amalg R\left(G_{2}\right)$. 
Next we prove the acyclicity of $\alpha\left(M_{i}^{j}\right)$. We have:

$$
\begin{aligned}
H\left(\alpha\left(M_{i}^{j}\right)\right) & =H\left(R\left(\left(F \Delta_{j} * G \bar{\Delta}_{i}\right) *{ }_{i} F \bar{\Delta}_{i}\right)\right) \\
& =H\left(R\left(F \Delta_{j} *\left(G \bar{\Delta}_{i} *{ }_{\tau} F \bar{\Delta}_{i}\right)\right)\right)=R .
\end{aligned}
$$

The second equality follows from the obvious isomorphism of the simplicial groups: $\left(F \Delta_{j} * G \bar{\Delta}_{i}\right) *{ }_{\bar{\tau}} F \bar{\Delta}_{i} \approx F \Delta_{j} *\left(G \bar{\Delta}_{i} *{ }_{\tau} F \bar{\Delta}_{i}\right)$. The third equality follows from the acyclicity of $F \Delta_{j}$ and $G \bar{\Delta}_{i} *{ }_{\tau} F \bar{\Delta}_{i}$, Theorem 1 of [6] and the remark in the beginning of this proof. $G \bar{\Delta}_{i}{ }^{*}{ }_{\tau} F \bar{\Delta}_{i}$ is acyclic because of the isomorphism $G \bar{\Delta}_{i}{ }{ }_{\tau} F \bar{\Delta}_{i} \approx G C \bar{\Delta}_{i}$ (C is the cone construction) and the fact that $G C \bar{\Delta}_{i}$ is contractible [8, 2.2.2, 1.3]. $F \Delta_{j}$ is acyclic because of the isomorphism $F \Delta_{j} \approx G S \Delta_{j}$ ( $S$ is the suspension construction), and the fact that $\Delta_{j}$ is contractible [8, 1.4]. (Here we used the commutativity in the order of applying loop, cone and suspension constructions with the geometric realization [7, Chapter III].)

The proof of the acyclicity of $\beta\left(M_{i}^{j}\right)$ is more involved. Consider the following map:

$$
k: R\left(F \Delta_{j}\right) \amalg\left(R\left(G \bar{\Delta}_{i}\right) \amalg_{f_{f}} T\left(R\left(\bar{\Delta}_{i}\right)\right)\right) \rightarrow R\left(F \Delta_{j} * G \bar{\Delta}_{i}\right) \amalg_{f_{f}} T\left(R\left(\bar{\Delta}_{i}\right)\right)
$$

where the first component is induced by the inclusion homomorphism $F \Delta_{j} \rightarrow$ $F \Delta_{j} * G \bar{\Delta}_{i}$ and the second component is induced by the map $\beta[v, u]$ where $v$ is the inclusion homomorphism $G \bar{\Delta}_{i} \rightarrow F \Delta_{j} * G \bar{\Delta}_{i}, u$ is the identity homomorphism of $F \bar{\Delta}_{i}$. If we filter both complexes by the degree of $T\left(R\left(\bar{\Delta}_{i}\right)\right), k$ will preserve the filtration. Since $T(R(X))$ is a free chain complex we have

$$
\begin{aligned}
k_{p, q}^{1}: \bar{E}_{p, q}^{1} & =H\left(R\left(F \Delta_{j}\right) \amalg R\left(G \bar{\Delta}_{i}\right)\right)_{q} \amalg_{p} T\left(R\left(\bar{\Delta}_{i}\right)\right) \rightarrow E_{p, q}^{1} \\
& =H\left(F \Delta_{j} * G \bar{\Delta}_{i}\right)_{q} \amalg_{p} T\left(R\left(\bar{\Delta}_{i}\right)\right),
\end{aligned}
$$

where $K_{q} \amalg_{p} L$ is the submodule of $(K \amalg L)_{p+q}$ whose elements are tensor products of elements of $K$ and $L$, where the sums of degrees of elements of $K$ and $L$ are $q$ and $p$ respectively. Since $k$ induces an isomorphism [6, Theorem 1]

$$
H\left(R\left(F \Delta_{j}\right) \amalg R\left(G \bar{\Delta}_{i}\right)\right) \rightarrow H\left(F \Delta_{j} * G \bar{\Delta}_{i}\right),
$$

$k$ induces an isomorphism $k^{1}: \bar{E}^{1} \rightarrow E^{1}$. Both spectral sequences are first quadrant spectral sequences and thus $k$ induces an isomorphism on the homology level.

Thus we must prove that $R\left(F \Delta_{j}\right) \amalg R\left(G \bar{\Delta}_{i}\right) \amalg_{f_{\tau}} T\left(R\left(\bar{\Delta}_{i}\right)\right)$ is acyclic. We already know that $R\left(F \Delta_{j}\right)$ is acyclic. Again using the remark in the beginning of this proof, it is enough to prove that $R\left(G \bar{\Delta}_{i}\right) \amalg_{f_{\tau}} T\left(R\left(\bar{\Delta}_{i}\right)\right)$ is acyclic.

Consider the following diagram:

$$
\begin{array}{ccc}
R\left(G \bar{\Delta}_{i}\right) \amalg_{f_{\tau}} T\left(R\left(\bar{\Delta}_{i}\right)\right) & \stackrel{\Phi}{\rightarrow} & T\left(R\left(G \bar{\Delta}_{i}\right) \otimes_{f_{\tau}} R\left(\bar{\Delta}_{i}\right)\right) \\
& 1 \searrow^{\searrow} & \downarrow_{\Psi} \\
& & R\left(G \bar{\Delta}_{i}\right) \amalg_{f_{\tau}} T\left(R\left(\bar{\Delta}_{i}\right)\right)
\end{array}
$$


where $\Psi$ is the algebra homomorphism induced by the obvious chain maps:

$$
\begin{aligned}
\tilde{R}\left(G \bar{\Delta}_{i}\right) \otimes 1 & \rightarrow \tilde{R}\left(G \bar{\Delta}_{i}\right), \\
1 \otimes \tilde{R}\left(\bar{\Delta}_{i}\right) & \rightarrow \tilde{R}\left(\bar{\Delta}_{i}\right) \subset T\left(R\left(\bar{\Delta}_{i}\right)\right), \\
\tilde{R}\left(G \bar{\Delta}_{i}\right) \otimes \tilde{R}\left(\bar{\Delta}_{i}\right) & \rightarrow \tilde{R}\left(G \bar{\Delta}_{i}\right) \otimes T\left(R\left(\bar{\Delta}_{i}\right)\right) .
\end{aligned}
$$

$\Phi$ is not an algebra homomorphism, but a differential chain map. If we denote homogeneous generators of $\tilde{R}\left(G \bar{\Delta}_{i}\right)$ and $\tilde{R}\left(\bar{\Delta}_{i}\right)$ by $g_{j}$ and $x_{j}$ respectively, then generators of $R\left(G \bar{\Delta}_{i}\right) \amalg_{f_{\tau}} T\left(R\left(\bar{\Delta}_{i}\right)\right)$ are of the form:

$$
\begin{aligned}
x_{1}^{1} \otimes \cdots \otimes & x_{1}^{a_{1}} \otimes g_{2} \otimes x_{2}^{1} \otimes \cdots \otimes x_{2}^{a_{2}} \otimes g_{3} \otimes x_{3}^{1} \otimes \ldots \\
& g_{1} \otimes x_{1}^{1} \otimes \cdots \otimes x_{1}^{b_{1}} \otimes g_{2} \otimes x_{2}^{1} \otimes \cdots \otimes x_{2}^{b_{2}} \otimes g_{3} \otimes x_{3}^{1} \otimes \ldots
\end{aligned}
$$

where $a_{i}$ and $b_{i}$ are positive integers, and the representations of the generators may have any finite number of elements in them. $\Phi$ is defined as follows:

$$
\begin{gathered}
\Phi\left(x_{1}^{1} \otimes \cdots \otimes x_{1}^{a_{1}} \otimes g_{2} \otimes x_{2}^{1} \otimes \ldots\right)=\left(1 \otimes x_{1}^{1}\right) \otimes \cdots \otimes\left(1 \otimes x_{1}^{a_{1}}\right) \\
\otimes\left(g_{2} \otimes x_{2}^{1}\right) \otimes\left(1 \otimes x_{2}^{2}\right) \otimes \cdots \otimes\left(1 \otimes x_{2}^{a_{2}}\right) \otimes\left(g_{3} \otimes x_{3}^{1}\right) \otimes \ldots, \\
\Phi\left(g_{1} \otimes x_{1}^{1} \otimes \ldots \otimes x_{1}^{b_{1}} \otimes g_{2} \otimes x_{2}^{1} \otimes \ldots\right)=\left(g_{1} \otimes x_{1}^{1}\right) \otimes\left(1 \otimes x_{1}^{2}\right) \otimes \ldots \\
\otimes\left(1 \otimes x_{1}^{b_{1}}\right) \otimes\left(g_{2} \otimes x_{2}^{1}\right) \otimes\left(1 \otimes x_{2}^{2}\right) \otimes \ldots
\end{gathered}
$$

If the generator ends with $g_{j}$, then $\Phi$ of the generator ends with $\left(g_{j} \otimes 1\right)$. It is straightforward to check that $\Phi$ is a chain map, and makes the diagram commutative. Since $R\left(G \bar{\Delta}_{i}\right) \otimes_{f_{\tau}} R\left(\bar{\Delta}_{i}\right)$ is acyclic, [3, 8.2] or [7, 31.5], a multiple use of the Künneth formula provides us with the acyclicity of $T\left(R\left(G \bar{\Delta}_{i}\right) \otimes_{f_{r}}\right.$ $\left.R\left(\bar{\Delta}_{i}\right)\right)$ and thus $R\left(G \bar{\Delta}_{i}\right) \amalg_{f_{\tau}} T\left(R\left(\bar{\Delta}_{i}\right)\right)$ is acyclic. This completes the proof of the acyclicity of $\beta\left(M_{i}^{j}\right)$ and completes the proof of Lemma 5.

Next, let us filter $R\left(A *{ }_{t} F X\right)$ and $R(A) \amalg_{f_{t}} T(R(X))$ as follows: an element of $R(A *, F X)$ has filtration degree $<p$ if the elements of $X$ in it are degenerates of elements of degree $<p$, an element of $R(A) \amalg_{f_{t}} T(R(X))$ has filtration degree $<p$ if the elements of $R(X)$ in it have degree $<p$. It is not difficult to see that $\alpha$ and $\beta$ with these filtrations have representations mutually related in the following sense:

LEMMA 6. If $y \in F_{p}(\alpha(A *, F X))$, (has $p$ filtration) then in its representation it has a nonzero component only in terms $\alpha(M, u)$ with the property:

$\beta(u) \beta(M) \subset F_{p}\left(R(A) \amalg_{f_{i}} T(R(X))\right)$, where $M$ is a model and $u$ a morphism. $A$ similar requirement holds when the roles of $\alpha$ and $\beta$ are exchanged.

Applying the method of acyclic models we are led to:

THEOREM 1. There are filtration preserving chain maps $h: \alpha \rightarrow \beta, k: \beta \rightarrow \alpha$ such that $k \circ h$ and $h \circ k$ are chain homotopic to the identity maps via filtration preserving chain homotopies. The maps $h, k$ induce isomorphisms on the corresponding spectral sequences.

To complete the proof we have to define $h_{i}$ and $k_{i}, i=0,1$, and show that $D h_{1}=h_{0} d, d k_{1}=k_{0} D$. We make the following definitions keeping in mind that $x_{0}$ consists of a single element. 
Throughout the proof, let $a \in A_{0}, b \in A_{1}$ and $x \in X_{1}$.

We define $h_{0}$ on $(\alpha(A *, F X))_{0}$ by $h_{0}(a)=a$. It is clear that this map commutes with the augmentation maps.

The definition of $h_{1}$ is more complicated. First we define some auxiliary functions on $A_{1}$ and $X_{1}$ :

$$
\begin{aligned}
B(b) & =\partial_{1} b, \quad B(x)=1, \quad B\left(x^{-1}\right)=1, \\
M(b) & =b, \quad M(x)=t(x) \otimes x, \quad M\left(x^{-1}\right)=-x, \\
E(b) & =\partial_{0} b, \quad E(x)=t(x), \quad E\left(x^{-1}\right)=t(x)^{-1} .
\end{aligned}
$$

We define $h_{1}$ on the generators of $(R(A *, F X))_{1}$ by:

$$
\begin{aligned}
h\left(y_{1} \cdots y_{n}\right)= & M\left(y_{1}\right) \otimes E\left(y_{2}\right) \otimes E\left(y_{3}\right) \otimes \cdots \otimes E\left(y_{n}\right) \\
& +B\left(y_{1}\right) \otimes M\left(y_{2}\right) \otimes E\left(y_{3}\right) \otimes \cdots \otimes E\left(y_{n}\right) \\
& +B\left(y_{1}\right) \otimes B\left(y_{2}\right) \otimes M\left(y_{3}\right) \otimes E\left(y_{4}\right) \otimes \cdots \otimes E\left(y_{n}\right) \\
& +\cdots+B\left(y_{1}\right) \otimes B\left(y_{2}\right) \otimes \cdots \otimes B\left(y_{n-1}\right) \otimes M\left(y_{n}\right),
\end{aligned}
$$

where $y_{i} \in A_{1} \cup X_{1} \cup X_{1}^{-1}$ such that $y_{1} \cdots y_{n} \in A_{1} *(F X)_{1}$.

We prove by induction on the number of elements in a generator that $D h_{1}=h_{0} d$. For $n=1$ we distinguish between three cases:

$$
\begin{aligned}
D h_{1}(b) & =D(M(b))=D(b)=\partial_{0} b-\partial_{1} b=h_{0} d(b), \\
D h_{1}(x) & =D(M(x))=D(t(x) \otimes x) \\
& =t(x)\left(1-t^{-1}(x)\right)=t(x)-1=h_{0} d(x), \\
D h_{1}\left(x^{-1}\right) & =D\left(M\left(x^{-1}\right)\right)=D(-x) \\
& =-\left(1-t(x)^{-1}\right)=t(x)^{-1}-1=h_{0} d\left(x^{-1}\right) .
\end{aligned}
$$

Now we assume $D h_{1}=h_{0} d$ for generators with less than $n+1$ elements, and prove the identity for generators with $n+1$ elements.

$$
\begin{aligned}
D h_{1}\left(y_{1} \cdots y_{n+1}\right)=D( & M\left(y_{1}\right) \otimes E\left(y_{2}\right) \otimes \cdots \otimes E\left(y_{n+1}\right)+\ldots \\
& \quad B\left(y_{1}\right) \otimes \cdots \otimes B\left(y_{n-1}\right) \otimes M\left(y_{n}\right) \otimes E\left(y_{n+1}\right) \\
& \left.+B\left(y_{1}\right) \otimes \cdots \otimes B\left(y_{n}\right) \otimes M\left(y_{n+1}\right)\right) \\
= & D\left(h_{1}\left(y_{1} \cdots y_{n}\right)\right) \otimes E\left(y_{n+1}\right)-h_{1}\left(y_{1} \cdots y_{n}\right) \otimes D\left(E y_{n+1}\right) \\
& +D\left(B\left(y_{1}\right) \otimes \cdots \otimes B\left(y_{n}\right) \otimes M\left(y_{n+1}\right)\right) \\
= & \left(h_{0} d\left(y_{1} \cdots y_{n}\right)\right) \otimes E\left(y_{n+1}\right) \\
& +B\left(y_{1}\right) \otimes \cdots \otimes B\left(y_{n}\right) \otimes D\left(M\left(y_{n+1}\right)\right) \\
= & h_{0}\left(d\left(y_{1} \cdots y_{n}\right) \cdot E\left(y_{n+1}\right)\right) \\
& +B\left(y_{1}\right) \otimes \cdots \otimes B\left(y_{n}\right) \otimes D\left(M\left(y_{n+1}\right)\right) .
\end{aligned}
$$

If $y_{n+1}=b$, then, $E\left(y_{n+1}\right)=\partial_{0} b, D\left(M\left(y_{n+1}\right)\right)=\partial_{0} b-\partial_{1} b$ and

$$
\begin{aligned}
B\left(y_{1}\right) \otimes & \cdots \otimes B\left(y_{n}\right) \otimes D\left(M\left(y_{n+1}\right)\right) \\
& =h_{0}\left(B\left(y_{1}\right) \cdots B\left(y_{n}\right) \cdot \partial_{0} b\right)-h_{0}\left(B\left(y_{1}\right) \cdots B\left(y_{n}\right) \cdot \partial_{1} b\right) .
\end{aligned}
$$


Substituting, we obtain the desired equality.

$$
\begin{aligned}
& \text { If } y_{n+1}=x \text {, then } E\left(y_{n+1}\right)=t(x), \\
& \qquad \begin{aligned}
D\left(M\left(y_{n+1}\right)\right) & =D(t(x) \otimes x)=t(x)\left(1-t(x)^{-1}\right)=t(x)-1, \\
h_{0}\left(d\left(y_{1} \cdots y_{n}\right)\right. & \left.E\left(y_{n+1}\right)\right) \\
& =h_{0}\left(\partial_{0} y_{1} \cdots \partial_{0} y_{n} \partial_{0} y_{n+1}\right)-h_{0}\left(\partial_{1} y_{1} \cdots \partial_{1} y_{n} \cdot t x\right)
\end{aligned}
\end{aligned}
$$

and

$$
\begin{aligned}
B\left(y_{1}\right) \otimes \cdots & \otimes B\left(y_{n}\right) \otimes D\left(M\left(y_{n+1}\right)\right) \\
& =h_{0}\left(\partial_{1} y_{1} \cdots \partial_{1} y_{n} \cdot t(x)\right)-h_{0}\left(\partial_{1} y_{1} \cdots \partial_{1} y_{n} \cdot \partial_{1} y_{n+1}\right) .
\end{aligned}
$$

Substituting, we again arrive at the desired equality. Last we assume that $y_{n+1}=x^{-1}$ and go through the above process and once more find that $D h_{1}=h_{0} d$.

$k_{0}$ is defined by requiring that $k_{0}(a)=a$. It is immediate that $k_{0}$ commutes with the augmentation maps. We define $k_{1}$ on 1-dimensional generators of $R(A) \amalg_{t_{f}} T(R(X))$. Let $z_{1} \otimes \cdots \otimes z_{n}$ be a generator of $R(A) \amalg_{f_{t}} T(R(X))_{1}$. Then, only one of the $z_{i}$, for example $z_{i_{0}}$, has dimension 1 , all the others have dimension 0. For $z_{i_{0}}=b \in A_{1}$ we define:

$$
k_{1}\left(z_{1} \otimes \cdots \otimes z_{n}\right)=s_{0} z_{1} \cdots s_{0} z_{i_{0}-1} \cdot b \cdot s_{0} z_{i_{0}+1} \cdots s_{0} z_{n}
$$

and for $z_{i_{0}}=x$ we define:

$$
k_{1}\left(z_{1} \otimes \cdots \otimes z_{n}\right)=s_{0} z_{1} \cdots s_{0} z_{i_{0}-1} \cdot s_{0}\left(t(x)^{-1}\right) \cdot x \cdot s_{0} z_{i_{0}+1} \cdots s_{0} z_{n} .
$$

It is easy to confirm that $d k_{1}=k_{0} D$.

We completed the definitions of $h_{i}, k_{i}, i=0,1$ for elements of the form $A *, F X$. The definition for tuples is exactly the same provided that we apply the proper twisting function to $x$ according to the t.f.p., to which $x$ belongs.

5. Applications. We recall the following definitions $[3, \S 10]$. Let $t: X \rightarrow A$ be a twisting function. We say that $t$ is $n$-trivial if it is the constant function on $X_{1}, X_{2}, \ldots, X_{n}$. We say that the twisting cochain $f_{t}: R(X) \rightarrow R(A)$ is $n$-trivial if $f_{t}$ is constantly 0 on $R_{1}(X), R_{2}(X), \ldots, R_{n}(X)$. We note that if $t$ is $n$-trivial, so is $f_{t}[3$, p. 305].

If we filter $R(A) \amalg_{f_{t}} T(R(X))$ by the degree of $T(R(X))$, and if $f$ is $n$-trivial then $D-D_{0}$ reduces filtration by $(n+1)$, and thus the differentials of the spectral sequences corresponding to $D$ and $D_{f}$ differ only from the $n+1$ level and beyond. Incorporating the results of [3, Theorems 10.3 and 10.4] and the fact that $D$ is a derivation we get the following:

THeOREM 2. Let $A *, F X$ be a p.c.b. with $n$-trivial twisting function, $n>1$. Then we obtain a first quadrant spectral sequence with:

$$
\begin{aligned}
& E_{p, q}^{1}=H(A)_{q} \amalg_{p} T(R(X)), \\
& E_{p, q}^{2}=E_{p, q}^{3}=\cdots=E_{p, q}^{n+1}=H_{p}\left(H(A)_{q} \amalg T(R(X))\right)
\end{aligned}
$$

where $H(A){ }_{q} \amalg T(R(X))=\cup{ }_{i=0}^{\infty} H(A){ }_{q} \amalg_{i} T(R(X))$. 
TheOREM 3. Assuming the situation of Theorem 2 and one of the following additional conditions:

(a) the ring $R$ is a field,

(b) $H(X)$ is torsion free, then $E_{p, q}^{2}=E_{p, q}^{3}=\cdots=E_{p, q}^{n+1}=H(A)_{q} \amalg_{p} T(H(X))$.

Furthermore, $d^{n+1}$ is the derivation induced by the map defined on $u \in H(x)$ by $d^{n+1} u=k \cap u$ and on elements of $H(A)$ by zero, where $k \in$ $H^{n+1}\left(X, H_{n}(A)\right)$ is the transgression element of $(-1)^{n+1} \gamma$ in the associated principal bundle of $A \times_{t} X$, and $\gamma \in R_{n}\left(A, H_{n}(A)\right)$ is the fundamental cocycle.

This theory applies to the computation of the homology of a space of the form $\Omega\left(Y \cup_{g} C X\right)$ in the following way. Consider the "cofibration" sequence $Y \rightarrow Y \cup_{g} C X \rightarrow S X$ in the category of simplicial sets. For the definition of $S, C$, and the imbedding of $X$ in $C X$ refer to $[8,1.3]$. In the above sequence $g$ is a simplicial map from $X$ to $Y$, and $Y \cup_{g} C X$ is the complex obtained by identifying the elements $x \in X \subset C X$ with $g(x) \in Y$. The loop spaces of the above sequence yield the elements of a p.c.b. $G Y, G\left(Y \cup_{g} C X\right), G S X=F X$. In fact, it is easy to obtain an isomorphism $G\left(Y \cup_{g} C X\right) \approx G Y{ }^{*}{ }_{g \circ \tau} F X$ as was done in [8, 2.2.2]. We would like to point out that the p.c.b. associated with $G Y *{ }_{g \circ \tau} F X$, is induced from the universal p.c.b. which is associated with $G X *{ }_{\tau} F X$ by the map $G(g)$ (see [5]). The twisting cochain corresponding to $g \circ \tau$ is $G(g) \circ f_{\tau}[3$, p. 304] and is $n$-trivial under one of the following conditions:

(i) $g$ is constant on the first $n$ dimensions of $X$.

(ii) $g$ is homotopic to a map $h$ which is constant on the first $\boldsymbol{n}$ dimensions.

(iii) $X$ is $n$ connected.

(iv) $Y$ is $n$ connected.

This is because: (i) is obvious, (ii) follows from the fact that loop homotopic homomorphisms induce isomorphic p.c.b.'s, and that the loops of homotopic maps are loop homotopic [5], (iii) follows from (ii), (iv) follows from the fact that the adjoint functors $G$ and $\bar{W}$ (the classifying space functor) preserve homotopies [7, §27].

We conclude this paper by demonstrating the use of our theory for the computation of $H\left(\Omega\left(Y \cup_{g} C S^{n+1}\right)\right)$, where $Y$ is simply connected. The $E^{n+1}$ term of the spectral sequence obtained by Theorem 3 has the following form:

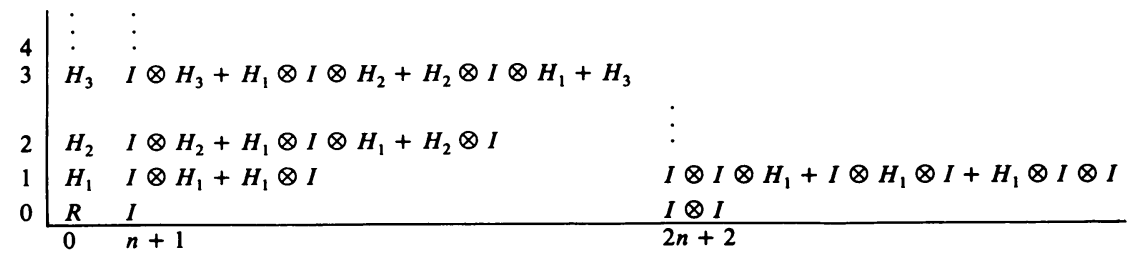

where $H_{i}=H_{i}(\Omega Y), I=H_{n+1}\left(S^{n+1}\right)$ and except for the columns which stand in $0(\bmod n+1)$ places everything else is 0 . In the case where $Y=S^{m}$, 
$m>1$, and $g$ is the constant map, we obtain the result of [6]. In the more general case we can deduce for example that $H_{i}\left(\Omega\left(Y \cup_{g} C S^{n+1}\right)\right) \approx H_{i}(\Omega Y)$ for $i=1,2, \ldots, n-1$, regardless of what specific map $g$ we take.

\section{REFERENCES}

1. I. Berstein, On co-groups in the category of graded algebras, Trans. Amer. Math. Soc. 115 (1965), 257-269. MR 34 \#6757.

2. E. H. Brown, Jr., Twisted tensor products. I, Ann. of Math. 6 (1959), 223-246.

3. V. K. A. M. Gugenheim, On a theorem of E. H. Brown, Illinois J. Math. 4 (1960), 292-311.

4. D. M. Kan, On moinoids and their dual, Bol. Soc. Mat. Mexicana 3 (1958), 52-61.

5. E. Katz, Twisted cartesian and free products, Atti Accad. Naz. Lincei Rend. Cl. Sci. Fis. Mat. Natur. 40 (1976), 235-239.

6. , A Künneth formula for coproducts of simplicial groups, Proc. Amer. Math. Soc. 61 (1976), 117-121.

7. J. P. May, Simplicial objects in algebraic topology, Van Nostrand, Princeton, N.J., 1967. MR 36 \#5942.

8. N. H. Schlomiuk, Principal cofibrations in the category of simplicial groups, Trans. Amer. Math. Soc. 146 (1969), 151-166.

9. L. Smith, Lectures on the Eilenberg-Moore spectral sequence, Lecture Notes in Math., vol. 134, Springer-Verlag, Berlin and New York, 1970.

Department of Mathematics, University of Haifa, Mt. Carmel, Haifa 31 999, Israei

Current address: Department of Mathematics, Tel-Aviv University, Tel-Aviv, Israel 